\title{
Clinicopathological features of colorectal polyps and risk of colorectal cancer in acromegaly
}

\author{
Yorinari Ochiaii,, Naoko Inoshita ${ }^{2,3,6}$, Toshiro lizuka ${ }^{1}$, Hiroshi Nishioka ${ }^{4}$, Shozo Yamada ${ }^{4,5}$, Masanobu Kitagawa \\ and Shu Hoteya ${ }^{1}$
}

${ }^{1}$ Department of Gastroenterology, Toranomon Hospital, Tokyo, Japan, ²Department of Pathology, Tokyo Metropolitan Geriatric Medical Center, Tokyo, Japan, ${ }^{3}$ Department of Pathology, Toranomon Hospital, Tokyo, Japan, ${ }^{4}$ Department of Hypothalamic and Pituitary Surgery, Toranomon Hospital, Tokyo, Japan, ${ }^{5}$ Department of Neurosurgery, Moriyama Memorial Hospital, Tokyo, Japan, and ${ }^{6}$ Department of Comprehensive Pathology, Graduate School of Medical and Dental Sciences, Tokyo Medical and Dental University, Tokyo, Japan

Correspondence should be addressed to Y Ochiai

Email

y.ochiai.1987@gmail.com

\section{Abstract}

Objective: Patients with acromegaly are at increased risk of colorectal polyps. However, their risk of colorectal cancer remains unclear. This study aimed to identify the histopathological features of colorectal polyps in patients with acromegaly and compare their risk of colorectal cancer with that in healthy controls.

Methods: The study participants were 178 patients who underwent Hardy's operation and perioperative colonoscopy at our hospital between April 2008 and September 2016. For the control group, we randomly selected 356 age- and sexmatched patients who underwent colonoscopy at our hospital during the same period. The incidence, size, location, and histology of the colorectal polyps detected were compared between the groups.

Results: Colorectal polyps were detected in $66.8 \%$ of the acromegaly group and $24.2 \%$ of the control group ( $P<0.001)$. The average number and size of the polyps were 2.44 and $4.74 \mathrm{~mm}$, respectively, in the acromegaly group and 1.77 and $3.89 \mathrm{~mm}$ in the control group $(P=0.001)$. Polyps in the acromegaly group were more likely to be in the rectosigmoid region $(P=0.006)$. In the acromegaly group, the frequency of polyps $\geq 5 \mathrm{~mm}$ was $34.3 \%$ and that for polyps $\geq 10 \mathrm{~mm}$ was $15.2 \%$; the respective values were $7.6 \%$ and $2.2 \%$ in the control group $(P<0.001)$. We found no evidence of between-group histopathological differences in the polyp specimens resected by endoscopy. Conclusions: Patients with acromegaly are at an increased risk of colorectal polyps, especially in the rectosigmoid region. However, there is no pathological evidence that they are at greater risk of colorectal cancer than the general population.

\section{Introduction}

Acromegaly is caused by a growth hormone-producing tumor in the pituitary gland and is associated with increased risk of cardiovascular, respiratory, metabolic, and neoplastic complications with higher mortality rates than in the general population. However, mortality in acromegaly has decreased and the cause of death in affected individuals has changed in recent years as a result of improved treatment. In recent reports, malignant tumors (35\%) were the most common cause of death, followed by heart disease and cerebrovascular disorders $(1,2)$. The high frequency of malignant tumors in the population with acromegaly has been attributed to the action of insulin-like growth factor, secretion of which is stimulated by growth hormone, a cell growth
Published by Bioscientifica Ltd. 
factor that may cause growth of these tumors. It has also been reported that acromegaly is associated with a high frequency of colorectal polyps and a high incidence of colorectal cancer. Therefore, clinical guidelines recommend regular surveillance colonoscopy. However, a recent review found no difference in the incidence of colorectal cancer between patients with acromegaly and the general population (3). These conflicting reports could be because the studies reported to date had small sample sizes and thus lacked adequate statistical power. There has been no study involving more than 150 cases at a single institution. The reviews available have analyzed reports for small numbers of cases and there have been no adequately matched single-center case-control studies. Therefore, although it is known that colorectal polyps are common in patients with acromegaly, whether these patients are at increased risk of colorectal malignancy is controversial.

The aim of this study was to determine the frequency of colorectal polyps in a large cohort of patients with acromegaly at a single institution. We also sought to determine if there was any pathological evidence that some polyps are non-neoplastic (e.g. hyperplastic) and others are colorectal adenomas or colon cancer.

\section{Subjects and methods}

\section{Patients}

In total, 749 patients who underwent surgery for acromegaly from April 2008 to September 2016 at our hospital were considered for enrollment in this retrospective matched case-control single-center study. We excluded 480 patients who either did not undergo colonoscopy or had no endoscopic data available and 91 who had undergone colonoscopy at other hospitals. After these exclusions, 178 patients in whom colonoscopy had been performed at our hospital were enrolled (Fig. 1). First, all patients without acromegaly who underwent colonoscopy during a check-up at our hospital during the same period were screened for eligibility to be enrolled as controls. Patients of either sex who were in the same age range as those in the acromegaly group were identified. Initially, one author numbered the patients randomly. Another author then randomly selected four times more patients than in the acromegaly group, as far as possible, and numbered these patients again. Finally, the other author randomly chose two numbers out of four per

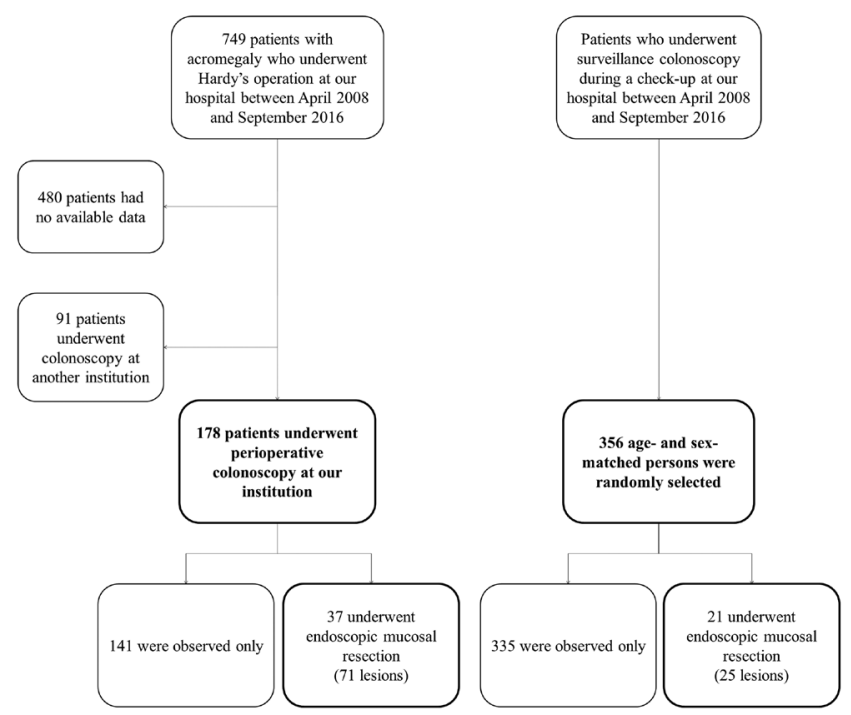

Figure 1

Flowchart of the study participant selection process.

patient (e.g. patients 1, 2, 3, and 4 were all male and aged 40 years, and the author chose No. 1 and 4). In this way, we randomly selected 356 patients matched for age and sex as a control group.

\section{Evaluation}

Demographic and anthropometric data, including weight and BMI, were collected retrospectively from the medical records. Information on the number, size, and location of the colonic polyps was extracted from endoscopic images and reports. The clinical features of the patients with polyps and the histopathological characteristics of the endoscopically resected polyp specimens were compared between the two groups.

\section{Statistical analysis}

The chi-squared test was used for between-group comparison of qualitative variables and the MannWhitney $U$ test for comparison of quantitative variables. All statistical analyses were performed using SPSS for Windows version 25.0 (IBM Corp.). A $P$-value of $<0.05$ was considered statistically significant.

\section{Ethics approval}

This study was approved by the ethics committee of Toranomon Hospital and was conducted in accordance with the Declaration of Helsinki. 


\section{Results}

\section{Demographic and anthropometric data}

The acromegaly group comprised of 84 men (47.2\%) and 94 women $(52.8 \%)$ with a median age of 47.5 (range, 18-75) years and the control group included 168 men and 188 women of median age 47.5 (23-75) years. There was a statistically significant difference in median height between the acromegaly group and the control group ( $165.7 \mathrm{~cm}$ vs $163.4 \mathrm{~cm} ; P=0.038$ ). There were also significant between-group differences in median body weight and BMI ( $65.1 \mathrm{~kg}$ and $23.4 \mathrm{~kg} / \mathrm{m}^{2}$ in the acromegaly groups and $60.1 \mathrm{~kg}$ and $22.5 \mathrm{~kg} / \mathrm{m}^{2}$ in the control group, respectively; $P<0.001$, Table 1 ).

\section{Characteristics of colorectal polyps}

The frequency of colorectal polyps was significantly higher in the acromegaly group than in the control group (66.8\% (119/178 cases) vs $24.2 \%$ ( $86 / 356$ cases); $P<0.001)$. Furthermore, the average number and size of the polyps was significantly larger in the acromegaly group than in the control group ( $P=0.001$, Table 2$)$. In the acromegaly group, polyps were located most often in the sigmoid colon and rectum (59\%). However, the proportion of polyps in the ascending colon and transverse colon was higher in the control group than in the acromegaly group. There was a significant difference in the proportion of colorectal polyps at these sites between the two groups $(P=0.006$; Table 3$)$.

The frequency of colorectal polyps was $34.3 \%$ (61/178 cases) in the acromegaly group and $7.6 \%$ (27/356 cases) in the control group when the polyp measured $\geq 5 \mathrm{~mm}$ and was $15.2 \%(27 / 178)$ and $2.2 \%$ (8/356 cases), respectively, when the polyp measured $\geq 10 \mathrm{~mm}$; the difference was significant regardless of size (both $P<0.001$; Table 4 ).

\section{Pathological findings}

A significantly greater number of endoscopic mucosal resection procedures were performed in the acromegaly group than in the control group $(20.8 \%$ vs $5.3 \%$, respectively; $P<0.001)$. Seventy-one specimens were endoscopically resected in the acromegaly group. Among these specimens were nine high-grade adenomas or adenocarcinomas (five adenocarcinomas: four mucosal cancers and one deep submucosal cancer (SM2)) in the acromegaly group. In contrast, only 25 lesions were resected in the control group, among them five high-grade adenomas or adenocarcinomas (two adenocarcinomas: one mucosal cancer and one shallow submucosal cancer (SM1)). There was no significant difference in the frequency of high-grade adenoma or adenocarcinoma between the groups. No other significant between-group pathological difference was observed (Table 5).

\section{Discussion}

The main cause of death in patients with acromegaly has recently shifted from cardiovascular disease to cancer (2). In Japan, the main cause of death in the general population in 2017 was cancer followed by heart disease (4), whereas in the European Union and the United States it was heart disease followed by cancer in both 2016 and $2017(5,6)$. However, due to decreasing rates of cardiovascular disease, several countries in the European Union are now recording a greater number of deaths from cancer (7). Based on these reports, the main causes of death in patients with acromegaly are similar to those of the general population in some countries.

It has been reported that patients with acromegaly are at an increased risk of colorectal tumors, particularly colorectal cancers $(8,9,10,11)$. Moreover, some reports suggest that the higher the blood growth hormone level,

Table 1 Study population characteristics. The data are shown as the median (range) or as the number as appropriate.

\begin{tabular}{|c|c|c|c|}
\hline & Acromegaly group & Control group & P-value \\
\hline Patients, $n$ & 178 & 356 & - \\
\hline Male:Female & $84: 94$ & $168: 188$ & - \\
\hline Age, years & $47.5(18-75)$ & $47.5(23-75)$ & - \\
\hline Height, cm & $165.7(145.5-191.4)$ & 163.4 (139.4-185) & 0.038 \\
\hline Weight, kg & $65.1(43.4-156)$ & $60.1(35.5-107.3)$ & $<0.001$ \\
\hline Body mass index, $\mathrm{kg} / \mathrm{m}^{2}$ & 23.4(17.3-43.2) & $22.5(15.1-37.2)$ & $<0.001$ \\
\hline $\mathrm{GH}, \mathrm{ng} / \mathrm{mL}$ & $11.5(0.6-541)$ & & - \\
\hline IGF-1, ng/mL & $626.5(205-1300)$ & & - \\
\hline
\end{tabular}


Table 2 Prevalence, number, and size of colorectal polyps detected in the acromegaly group and the control group.

Patients with polyps, $n$
Proportion of patients with polyps (\%)
Mean number of polyps
Mean size of polyps, mm

\begin{tabular}{c}
\hline Acromegaly group \\
\hline 119 \\
66.9 \\
$2.44 \pm 1.80$ \\
$4.74 \pm 5.00$ \\
\hline
\end{tabular}

\begin{tabular}{crr}
\hline Control group & & P-value \\
\hline 86 & & - \\
24.2 & & 0.001 \\
$1.77 \pm 1.11$ & 0.001 \\
$3.89 \pm 4.64$ & 0.001 \\
\hline
\end{tabular}

the greater the likelihood of developing colorectal cancer $(8$, 11). The current guidelines recommend that colonoscopy should be performed at the time of diagnosis of acromegaly and when affected individuals are over 40 years of age and that endoscopy should be performed regularly to screen for colorectal tumors $(12,13)$. Furthermore, a recent study found that growth hormone is necessary for growth of neoplasms in the colon (14). A review published in 2017 reported that there were insufficient data to suggest that the risk of colon cancer is higher in patients with acromegaly than in the general population (3). However, the previous reports were based on single-center studies containing fewer than 100 patients with acromegaly. Our study differs from the previous research in that it contains a high number of cases. Nevertheless, like previous researchers, we also found a larger number of polyps in patients with acromegaly than in the general population but no significant difference in histological types when pathological specimens obtained by endoscopic resection were reassessed. Of particular note was that there was no significant between-group difference in the rate of polyps that progressed to colorectal cancer. Therefore, the results of this study support the review published in 2017.

There have been a few reports on the anatomic location of colonic polyps in patients with acromegaly, which concluded that patients with acromegaly are more likely to develop right-sided colonic polyps (15, 16). However, we found significantly more polyps in

Table 3 Location of the colorectal polyps detected in the acromegaly group and the control group. A statistically difference of $P=0.006$ * was obtained whilst comparing the $n(\%)$ of colorectal polyps between the groups

\begin{tabular}{|c|c|c|}
\hline & \multicolumn{2}{|c|}{ Colorectal polyps in segment, $\boldsymbol{n}(\%)$} \\
\hline & Acromegaly group & Control group \\
\hline Cecum & $17(5.9)$ & $11(7.3)$ \\
\hline Ascending colon & $48(16.5)$ & $37(24.3)$ \\
\hline Transverse colon & $34(11.7)$ & $33(21.7)$ \\
\hline Descending colon & 20 (6.9) & $9(5.9)$ \\
\hline Sigmoid & $113(39.0)$ & $40(26.3)$ \\
\hline Rectum & $58(20.0)$ & $22(14.5)$ \\
\hline Sigmoid and rectum & $171(59.0)$ & $62(40.8)$ \\
\hline
\end{tabular}

*Does not include the Sigmoid and rectum. the sigmoid colon and rectum in the acromegaly group than in the control group, which is a new finding. Previous reports indicate that the colon in patients with acromegaly is about $20 \%$ longer than that in the general population and significantly more likely to form an endoscopic loop in the pelvis during colonoscope insertion. Moreover, insertion time has been reported to be significantly longer in patients with acromegaly than in the general population $(17,18)$. From these reports, it can be inferred that the distance between the sigmoid colon and rectum is particularly long in patients with acromegaly, rendering them likely to develop more polyps than the general population. Therefore, it is important to carefully observe the full length of the sigmoid colon and rectum when performing colonoscopy to check for polyps in these patients. Although screening colonoscopy will inevitably lead to an increase in the number of patients with acromegaly and colonic polyps, there are no data suggesting that early detection of polyps improves survival in this group. In this study, we only analyzed the pathological features of polyp specimens that had been resected by endoscopy. Therefore, whether or not the remaining small polyps affect the prognosis in patients with acromegaly is still unclear. Further studies are needed to clarify whether colonoscopy should be performed more frequently in patients with acromegaly than in the normal population.

This study has some limitations, which stem mainly from its retrospective single-center design. Also, we cannot exclude the possibility of a degree of selection bias in the groups. Most of the patients with acromegaly were referred to our institution for pituitary surgery and some might have already undergone preoperative screening colonoscopy, the results of which were not available to

Table 4 Prevalence of colorectal polyps measuring at least $5 \mathrm{~mm}$ and those measuring at least $10 \mathrm{~mm}$ in the acromegaly group and the control group.

\begin{tabular}{|c|c|c|c|}
\hline & Acromegaly group & Control group & $P$-value \\
\hline \multicolumn{4}{|c|}{$\begin{array}{l}\text { Patients with } \\
\text { polyps, } n(\%)\end{array}$} \\
\hline$\geq 5 \mathrm{~mm}$ & $61(34.3)$ & $25(7)$ & $<0.001$ \\
\hline$\geq 10 \mathrm{~mm}$ & $27(15.2)$ & $8(2.2)$ & $<0.001$ \\
\hline
\end{tabular}


Table 5 Histopathological features of colorectal polyps in the acromegaly group and the control group.

\begin{tabular}{lcc}
\hline & Acromegaly \\
\hline Number of polyps & 290 \\
Number of EMRs & 71 \\
Number of EMR performed patients (\%) & $37(20.8)$ \\
Hyperplastic polyp & 8 \\
SSA/P & 2 \\
Inflammatory polyp & 3 \\
Carcinoid & 1 \\
Low-grade adenoma & 48 \\
High-grade adenoma - Cancer & 9 \\
Cancer & 5 \\
\hline
\end{tabular}

\begin{tabular}{c}
\hline Control \\
\hline 152 \\
25 \\
$19(5.3)$ \\
1 \\
0 \\
0 \\
0 \\
19 \\
5 \\
2 \\
\hline
\end{tabular}

\begin{tabular}{c}
\hline P-value \\
\hline- \\
- \\
$<0.001$ \\
0.439 \\
1.00 \\
0.567 \\
1.00 \\
0.384 \\
0.344 \\
1.00
\end{tabular}

EMR, Endoscopic mucosal resection; SSA/P, Sessile serrated adenoma/polyp.

us at our institution. Finally, the acromegaly and control groups were matched for only age and sex, and so it is possible that there were unadjusted differences in other background factors.

\section{Conclusion}

In this study, we found a significantly greater number of colorectal polyps in patients with acromegaly than in controls matched for age and sex. These polyps were often found between the rectum and sigmoid colon. However, there was no difference in the pathological diagnosis of these polyps between patients with and without acromegaly. Our findings suggest that patients with acromegaly are not at an increased risk of colorectal cancer.

\section{Declaration of interest}

The authors declare that there is no conflict of interest that could be perceived as prejudicing the impartiality of this study.

\section{Funding}

This research did not receive any specific grant from any funding agency in the public, commercial, or not-for-profit sector.

\section{References}

1 Ritvonen E, Loyttyniemi E, Jaatinen P, Ebeling T, Moilanen L, Nuutila P, Kauppinen-Makelin R \& Schalin-Jantti C. Mortality in acromegaly: a 20-year follow-up study. Endocrine-Related Cancer 2016 23 469-480. (https://doi.org/10.1530/ERC-16-0106)

2 Bolfi F, Neves AF, Boguszewski CL \& Nunes-Nogueira VS. Mortality in acromegaly decreased in the last decade: a systematic review and meta-analysis. European Journal of Endocrinology 2018179 59-71. (https://doi.org/10.1530/EJE-18-0255)

3 Tirosh A \& Shimon I. Complications of acromegaly: thyroid and colon. Pituitary 201720 70-75. (https://doi.org/10.1007/s11102-0160744-z)
4 Japanese Ministry of Health, Labour and Welfare. Analysis by cause of death, 2017. (available at: https://www.mhlw.go.jp/english/ database/db-hw/lifetb17/dl/lifetb17-04.pdf)

5 Eurostat. Cause of death statistics, 2016. (available at: https:// ec.europa.eu/eurostat/statistics-explained/index.php?title=Causes_ of_death_statistics)

6 Centers for Disease Control and Prevention. Leading causes of death, 2017. (available at: https://www.cdc.gov/nchs/fastats/leading-causesof-death.htm)

7 Townsend N, Wilson L, Bhatnagar P, Wickramasinghe K, Rayner M $\&$ Nichols M. Cardiovascular disease in Europe: epidemiological update 2016. European Heart Journal 201637 3232-3245. (https://doi. org/10.1093/eurheartj/ehw334)

8 Yamamoto M, Fukuoka H, Iguchi G, Matsumoto R, Takahashi M, Nishizawa H, Suda K, Bando H \& Takahashi Y. The prevalence and associated factors of colorectal neoplasms in acromegaly: a single center based study. Pituitary 201518 343-351. (https://doi. org/10.1007/s11102-014-0580-y)

9 Kurimoto M, Fukuda I, Hizuka N \& Takano K. The prevalence of benign and malignant tumors in patients with acromegaly at a single institute. Endocrine Journal 200855 67-71. (https://doi.org/10.1507/ endocrj.k07e-010)

10 Koksal AR, Ergun M, Boga S, Alkim H, Bayram M, Altuntas Y, Ozguven Yilmaz B \& Alkim C. Increased prevalence of colorectal polyp in acromegaly patients: a case-control study. Diagnostic and Therapeutic Endoscopy 20142014 152049. (https://doi. org/10.1155/2014/152049)

11 Matano Y, Okada T, Suzuki A, Yoneda T, Takeda Y \& Mabuchi H. Risk of colorectal neoplasm in patients with acromegaly and its relationship with serum growth hormone levels. American Journal of Gastroenterology 2005100 1154-1160. (https://doi.org/10.1111/ j.1572-0241.2005.40808.x)

12 Dworakowska D, Gueorguiev M, Kelly P, Monson JP, Besser GM, Chew SL, Akker SA, Drake WM, Fairclough PD, Grossman AB et al. Repeated colonoscopic screening of patients with acromegaly: 15-year experience identifies those at risk of new colonic neoplasia and allows for effective screening guidelines. European Journal of Endocrinology 2010163 21-28. (https://doi.org/10.1530/EJE-091080)

13 Lois K, Bukowczan J, Perros P, Jones S, Gunn M \& James RA. The role of colonoscopic screening in acromegaly revisited: review of current literature and practice guidelines. Pituitary 201518 568-574. (https:// doi.org/10.1007/s11102-014-0586-5)

14 Chesnokova V, Zonis S, Zhou C, Recouvreux MV, Ben-Shlomo A, Araki T, Barrett R, Workman M, Wawrowsky K, Ljubimov VA et al. Growth hormone is permissive for neoplastic colon growth. PNAS 2016113 E3250-E3259. (https://doi.org/10.1073/ pnas.1600561113) 
15 Delhougne B, Deneux C, Abs R, Chanson P, Fierens H, LaurentPuig P, Duysburgh I, Stevenaert A, Tabarin A, Delwaide J et al. The prevalence of colonic polyps in acromegaly: a colonoscopic and pathological study in 103 patients. Journal of Clinical Endocrinology and Metabolism 199580 3223-3226. (https://doi.org/10.1210/ jcem.80.11.7593429)

16 Renehan AG, Bhaskar P, Painter JE, O’Dwyer ST, Haboubi N, Varma J, Ball SG \& Shalet SM. The prevalence and characteristics of colorectal neoplasia in acromegaly. Journal of Clinical Endocrinology and Metabolism 200085 3417-3424. (https://doi.org/10.1210/jcem.85.9.6775)
17 Renehan AG, Painter JE, Bell GD, Rowland RS, O’Dwyer ST \& Shalet SM. Determination of large bowel length and loop complexity in patients with acromegaly undergoing screening colonoscopy. Clinical Endocrinology 200562 323-330. (https://doi.org/10.1111/ j.1365-2265.2005.02217.x)

18 Iwamuro M, Yasuda M, Hasegawa K, Fujisawa S, Ogura-Ochi K, Sugihara Y, Harada K, Hiraoka S, Okada H \& Otsuka F. Colonoscopy examination requires a longer time in patients with acromegaly than in other individuals. Endocrine Journal 201865 151-157. (https://doi. org/10.1507/endocrj.EJ17-0322)

Received 12 October 2019

Revised version received 10 January 2020

Accepted 15 January 2020 